\title{
An integrated clinico-transcriptomic approach identifies a central role of the heme degradation pathway for septic complications after trauma
}

Rittirsch, Daniel ; Schoenborn, Veit ; Lindig, Sandro ; Wanner, Elisabeth ; Sprengel, Kai ; Günkel, Sebastian ; Blaess, Markus ; Schaarschmidt, Barbara ; Sailer, Patricia ; Märsmann, Sonja ; Simmen, Hans-Peter ; Cinelli, Paolo ; Bauer, Michael ; Claus, Ralf A ; Wanner, Guido A

\begin{abstract}
OBJECTIVE: The present study was aimed to identify mechanisms linked to complicated courses and adverse events after severe trauma by a systems biology approach. SUMMARY BACKGROUND DATA: In severe trauma, overwhelming systemic inflammation can result in additional damage and the development of complications, including sepsis. METHODS: In a prospective, longitudinal single-center study, RNA samples from circulating leukocytes from patients with multiple injury (injury severity score 17 points; $\mathrm{n}=81$ ) were analyzed for dynamic changes in gene expression over a period of 21 days by whole-genome screening (discovery set; $\mathrm{n}=10$ patients; 90 samples) and quantitative RTPCR (validation set; $\mathrm{n}=71$ patients, 517 samples). Multivariate correlational analysis of transcripts and clinical parameters was used to identify mechanisms related to sepsis. RESULTS: Transcriptome profiling of the discovery set revealed the strongest changes between patients with either systemic inflammation or sepsis in gene expression of the heme degradation pathway. Using quantitative RT-PCR analyses (validation set), the key components haptoglobin (HP), cluster of differentiation (CD) 163, heme oxygenase-1 (HMOX1), and biliverdin reductase A (BLVRA) showed robust changes following trauma. Upregulation of HP was associated with the severity of systemic inflammation and the development of sepsis. Patients who received allogeneic blood transfusions had a higher incidence of nosocomial infections and sepsis, and the amount of blood transfusion as source of free heme correlated with the expression pattern of HP. CONCLUSIONS: These findings indicate that the heme degradation pathway is associated with increased susceptibility to septic complications after trauma, which is indicated by HP expression in particular.
\end{abstract}

DOI: https://doi.org/10.1097/SLA.0000000000001553

Posted at the Zurich Open Repository and Archive, University of Zurich

ZORA URL: https://doi.org/10.5167/uzh-118242

Journal Article

Published Version

Originally published at:

Rittirsch, Daniel; Schoenborn, Veit; Lindig, Sandro; Wanner, Elisabeth; Sprengel, Kai; Günkel, Sebastian; Blaess, Markus; Schaarschmidt, Barbara; Sailer, Patricia; Märsmann, Sonja; Simmen, HansPeter; Cinelli, Paolo; Bauer, Michael; Claus, Ralf A; Wanner, Guido A (2016). An integrated clinicotranscriptomic approach identifies a central role of the heme degradation pathway for septic complications after trauma. Annals of Surgery, 264(6):1125-1134.

DOI: https://doi.org/10.1097/SLA.0000000000001553 


\title{
An Integrated Clinico-transcriptomic Approach Identifies a Central Role of the Heme Degradation Pathway for Septic Complications After Trauma
}

\author{
Daniel Rittirsch, MD, ${ }^{*}$ Veit Schoenborn, MD, ${ }^{*}$ Sandro Lindig, PhD, $\dagger$ Elisabeth Wanner, MD, ${ }^{*}$ \\ Kai Sprengel, MD, ${ }^{*}$ Sebastian Günkel, MD, ${ }^{*}$ Markus Blaess, PhD, $\dagger \dagger$ Barbara Schaarschmidt, MSc, $\dagger \ddagger$ \\ Patricia Sailer, ${ }^{*}$ Sonja Märsmann, ${ }^{*}$ Hans-Peter Simmen, MD, ${ }^{*}$ Paolo Cinelli, PhD, ${ }^{*}$ \\ Michael Bauer, MD, $\dagger \ddagger$ Ralf A. Claus, PhD, $\dagger \ddagger$ and Guido A. Wanner, MD*
}

\begin{abstract}
Objective: The present study was aimed to identify mechanisms linked to complicated courses and adverse events after severe trauma by a systems biology approach.

Summary Background Data: In severe trauma, overwhelming systemic inflammation can result in additional damage and the development of complications, including sepsis.

Methods: In a prospective, longitudinal single-center study, RNA samples from circulating leukocytes from patients with multiple injury (injury severity score $\geq 17$ points; $n=81$ ) were analyzed for dynamic changes in gene expression over a period of 21 days by whole-genome screening (discovery set; $\mathrm{n}=10$ patients; 90 samples) and quantitative RT-PCR (validation set; $\mathrm{n}=71$ patients, 517 samples). Multivariate correlational analysis of transcripts and clinical parameters was used to identify mechanisms related to sepsis.

Results: Transcriptome profiling of the discovery set revealed the strongest changes between patients with either systemic inflammation or sepsis in gene expression of the heme degradation pathway. Using quantitative RT-PCR analyses (validation set), the key components haptoglobin (HP), cluster of differentiation (CD) 163, heme oxygenase-1 (HMOX1), and biliverdin reductase A (BLVRA) showed robust changes following trauma. Upregulation of HP was associated with the severity of systemic inflammation and the development of sepsis. Patients who received allogeneic blood transfusions had a higher incidence of nosocomial infections and sepsis, and the amount of blood transfusion as source of free heme correlated with the expression pattern of HP. Conclusions: These findings indicate that the heme degradation pathway is associated with increased susceptibility to septic complications after trauma, which is indicated by HP expression in particular.
\end{abstract}

From the ${ }^{*}$ Division of Trauma Surgery, Department of Surgery, University Hospital Zurich, University of Zurich, Zurich, Switzerland; †Department of Anaesthesiology and Intensive Care Therapy, Jena University Hospital, Jena, Germany; and $\ddagger$ Center for Sepsis Control and Care, Jena University Hospital, Jena, Germany.

All authors contributed equally.

All authors jointly supervised this study.

This work was partly supported by grants from the Stiftung für Wissenschaftliche Forschung of the University of Zurich, the German Federal Ministry of Education and Research (BMBF), grant 01EO1002 to the Center for Sepsis Control and Care, grant 03Z2J521 to Meta-ZIK, and from the German Research Foundation DFG, Forschergruppe 1738, hhdp - heme and heme degradation products, alternative functions, and mechanisms.

Supplemental digital content is available for this article. Direct URL citations appear in the printed text and are provided in the HTML and PDF versions of this article on the journal's Web site (www.annalsofsurgery.com).

The authors report no conflicts of interest.

Reprints: Guido A. Wanner, Division of Trauma Surgery, Department of Surgery, University Hospital Zurich, Raemistrasse 100, CH-8091 Zurich, Switzerland. E-mail: guido.wanner@usz.ch.

Copyright (C) 2015 Wolters Kluwer Health, Inc. All rights reserved.

ISSN: 0003-4932/14/26105-0821

DOI: $10.1097 /$ SLA.0000000000001553
Keywords: heme degradation pathway, multiple injury, sepsis, systemic inflammation, transcriptome profiling, trauma

(Ann Surg 2015;xx:xxx-xxx)

$M$ ultiple trauma still represents one of the leading causes of death in western countries, and treatment of severely injured patients imposes a considerable burden for healthcare systems. ${ }^{1-3}$ Severe trauma triggers systemic inflammation, the clinical presentation of which is indistinguishable from sepsis syndrome, but not necessarily associated with infection. ${ }^{4,5}$ Already decades ago, it has been demonstrated that multiorgan failure (MOF) frequently occurred in trauma patients without infection. ${ }^{6}$ While the overall mortality rate from multiple trauma has declined significantly, the incidence of secondary sepsis after trauma remained unchanged during the past decade and represents a dreaded complication with prolonged necessity of intensive care and an unfavorable outcome. ${ }^{1}$ The incidence of MOF induced by traumatic shock has decreased substantially, whereas a decrease in MOF incidence due to sepsis is not observed. ${ }^{1}$ The decrease in inhospital mortality from severe trauma was inter alia achieved because the underlying pathophysiology is reflected by current treatment concepts, including damage control surgery and evidence-based protocols for fluid resuscitation, blood transfusion, and the management of traumatic coagulopathy. ${ }^{7-9}$ With the definition of sepsis criteria in the 1990 s, it has been conceptualized that the immune response after trauma follows a biphasic course with an early hyperinflammation (systemic inflammatory response syndrome; SIRS) that is terminated by immunosuppression during the later course (compensatory antiinflammatory response syndrome). ${ }^{10,11}$ In this concept, MOF was thought to be either induced by an overwhelming initial insult ("onehit model") or SIRS was amplified by a second insult (eg, surgical intervention; "two-hit model"). 5,10,12 Recently published findings of the Glue Grant Consortium on leukocyte genomic expression patterns showed the simultaneous induction of a plethora of pro- and anti-inflammatory genes after trauma, whereas adaptive immunityassociated genes were found to be suppressed. Based on these data, a novel model ("genomic storm") has been derived, which challenges the second hit theory of the traditional SIRS-compensatory antiinflammatory response syndrome paradigm, as the response of patients with different courses after trauma was found to be more common than different. ${ }^{5,13}$ Although recent research has improved our understanding of the network of systemic inflammation, the mechanisms that lead to adverse events, including nosocomial infections, MOF, and sepsis are inadequately understood, and specific immune-modulatory therapies are still far from reach for the treatment of trauma patients. ${ }^{12,14}$ 
In the present study, we aimed to investigate mechanisms of systemic inflammation after severe trauma by analysis of leukocyte transcriptomic expression patterns in order to identify pathways that are associated with infectious complications and sepsis, in particular.

\section{METHODS}

\section{Study Design}

Blood was sampled from 104 patients with multi-system trauma admitted to the Division of Trauma Surgery (level I trauma center) at the University Hospital Zurich from 12/2009 to 03/2012. Criteria for study enrollment included patient age $\geq 18$ years, an Injury Severity Score (ISS) $\geq 17$ points, and time from injury to admission $<6$ hours. All patients were recruited into the study under informed consent guidelines approved by the Local Ethical Committee (StV 26-2007) and international ethical guidelines (ClinicalTrials.gov-Identifier: NCT02508272). Study subjects were treated under the guidance of standard operating procedures developed and implemented at the University Hospital Zurich (based on guidelines of the German Society of Trauma; DGU). ${ }^{15}$ Whole blood from trauma patients was collected within the first 6 hours after trauma (day 0) and on days 1, 2, 3, 5, 7, 10, 14, and 21. Clinical outcomes and complications within 28 days after injury were recorded. Patient enrollment and study design are illustrated in Supplemental Fig. 1, http://links.lww.com/SLA/A929. For discovery, 10 representative patients (discovery set; $n=90$ samples) were selected based on their clinical presentation with respect to the development of secondary sepsis ( $\mathrm{n}=5$ patients; $\mathrm{n}=45$ samples) or systemic inflammation without infection ( $n=5$ patients; $n=45$ samples) for whole-genome screening. After statistical gene selection, results were validated in the remaining patients of the total cohort (validation set; $n=71$ patients; $n=517$ samples) by quantitative RT-PCR.

\section{Clinical Data}

Clinical data were prospectively collected in parallel with the corresponding blood samples. The occurrence and severity of systemic inflammation, sepsis, MOF, and nosocomial infections were retrospectively analyzed using the corresponding clinical parameters from patients' records. MOF was defined according to the Sequential Organ Failure Assessment (SOFA) score. ${ }^{16}$ Systemic inflammation and sepsis was defined according to criteria of the current guidelines. ${ }^{17-19}$ For assessment of the severity of traumainduced systemic inflammation and to define secondary sepsis in trauma patients a scoring system has been applied (SI score; for details see Supplemental Information, Supplemental Table 1 and Supplemental Fig. 2, http://links.lww.com/SLA/A929). ${ }^{20-24}$

\section{RNA Isolation}

PaxGene (PreAnalytix, Hombrechtikon, Switzerland) tubes were used for sampling and preservation of whole blood, and total cellular RNA from circulating leukocytes was isolated (PaxGene Blood RNA Kit; PreAnalytix) in a Qiacube apparatus according to the manufacturer's instructions. RNA integrity was proven using Experion (Biorad, Munich, Germany) microcapillary electrophoresis. A detailed description of the totRNA quality control and sample selection criteria is provided in Supplement, http://links.lww.com/ SLA/A929. For cDNA-synthesis, $1 \mu \mathrm{g}$ total RNA per sample was transcribed (RevertAid First Strand cDNA Synthesis, ThermoFisher Scientific; PTC-200 Thermal Cycler Dual, BioRad) according to manufacturer's protocol.

\section{Microarray Analysis}

A total of $200 \mathrm{ng}$ RNA of each sample was reversely transcribed and amplified using the TargetAmp-Nano Labeling Kit for
Illumina Expression BeadChips (Biozym Scientific GmbH, Hessisch Oldendorf, Germany) according to the manufacturer's instructions on a Biorad MJ Thermal Cycler (Biorad). All cRNA samples were purified the NucleoSpin RNA Clean-up system (Macherey-Nagel, GmbH \& Co. KG, Düren, Germany) and quantified using a NanoDrop spectrophotometer ND-2000 (Thermo Fisher Scientific, Schwerte, Germany/PEQLAB Biotechnologie, Carlsbad, CA) before proceeding to sample hybridization. Samples were prepared and hybridized on Illumina Human-HT-12-V4 Expression BeadChips (Illumina, San Diego, CA, USA; for details see Supplemental Information, http://links.lww.com/SLA/A929). For posthybridization data read-out and data preprocessing, including spot detection, gene mapping, and averaging of replicates, iScan Control Software was used. Control probe quality check was performed using Illumina Genome Studio-Software.

Statistical analysis (for details see Supplement, http://links. lww.com/SLA/A929) was performed using R software (http:// www.r-project.org/) and packages from Bioconductor. ${ }^{25}$ Differentially expressed genes were filtered by exhibiting above medianaveraged 1.5-fold change, estimate of significance $P \leq 0.05$ and a false discovery rate $<0.05$. Candidate genes selected for further analysis and validation by quantitative real-time PCR were identified by gene-set enrichment analysis (list is provided in Supplemental Table 5, http://links.lww.com/SLA/A929).

\section{Quantitative RT-PCR}

RT-qPCR was performed in a two-step protocol using RotorGene system and Rotor-Gene SYBR Green PCR Kit (Qiagen, Hilden, Germany) according to manufacturer's information with $250 \mathrm{nM}$ Primer mix and $25 \mathrm{ng} \mathrm{cDNA}$. Initial denaturation at $95^{\circ} \mathrm{C}$ for 5 minutes, followed by 50 cycles of denaturation at $95^{\circ} \mathrm{C}$ for 5 seconds and annealing/extension at a given temperature (see table) for 15 seconds, finally followed by a melting curve. Ct values and efficiency were documented for each sample, data were normalized to unchanged transcripts, whereby housekeeper adjustment $(\Delta \mathrm{Ct})$ was performed via expressed ACTB $[\Delta \mathrm{Ct}=\mathrm{Ct}(\mathrm{ACTB})$ - $\mathrm{Ct}$ (Candidate)]. The primer sequences are listed in Supplemental Table 2, http://links.lww.com/SLA/A929. No Ct values of genes with significant increases/decreases as well as reference genes included in this study were detected above 26 cycles. Comparisons of PCR values for the various groups are displayed in Box-and-Whisker plots. Significance was attained at $P<0.05$ using the Mann-Whitney $U$ (Wilcoxon rank sum) test. Similarity was assessed using parametric $(r)$ and nonparametric $(\rho)$ measures. Influences of clinical variables and their interactions on gene expression were investigated using multivariate ANOVA.

\section{RESULTS}

\section{Transcriptome Profiling for Hypothesis Generation}

Characteristics of the patient cohort are displayed in Table 1 (for further details see Supplemental Information and Supplemental Table 3, http://links.lww.com/SLA/A929).

In a discovery set, RNA samples from 10 representative trauma patients (all time points) diagnosed with either secondary sepsis or systemic inflammation without infection, respectively, were analyzed by whole-genome screening of differential gene expression patterns. Using unsupervised clustering, patients with systemic inflammation only and sepsis patients showed a distinct expression pattern and the discrimination of clinical presentation (Fig. 1A). Explorative gene-set analysis revealed robust upregulation of genes related to "hemoglobin metabolism/oxygen transport" and "pathogenic Escherichia coli infection," whereas the strongest downregulation was found for "ribosome-associated genes" (Table 2). 
TABLE 1. Patient Characteristics

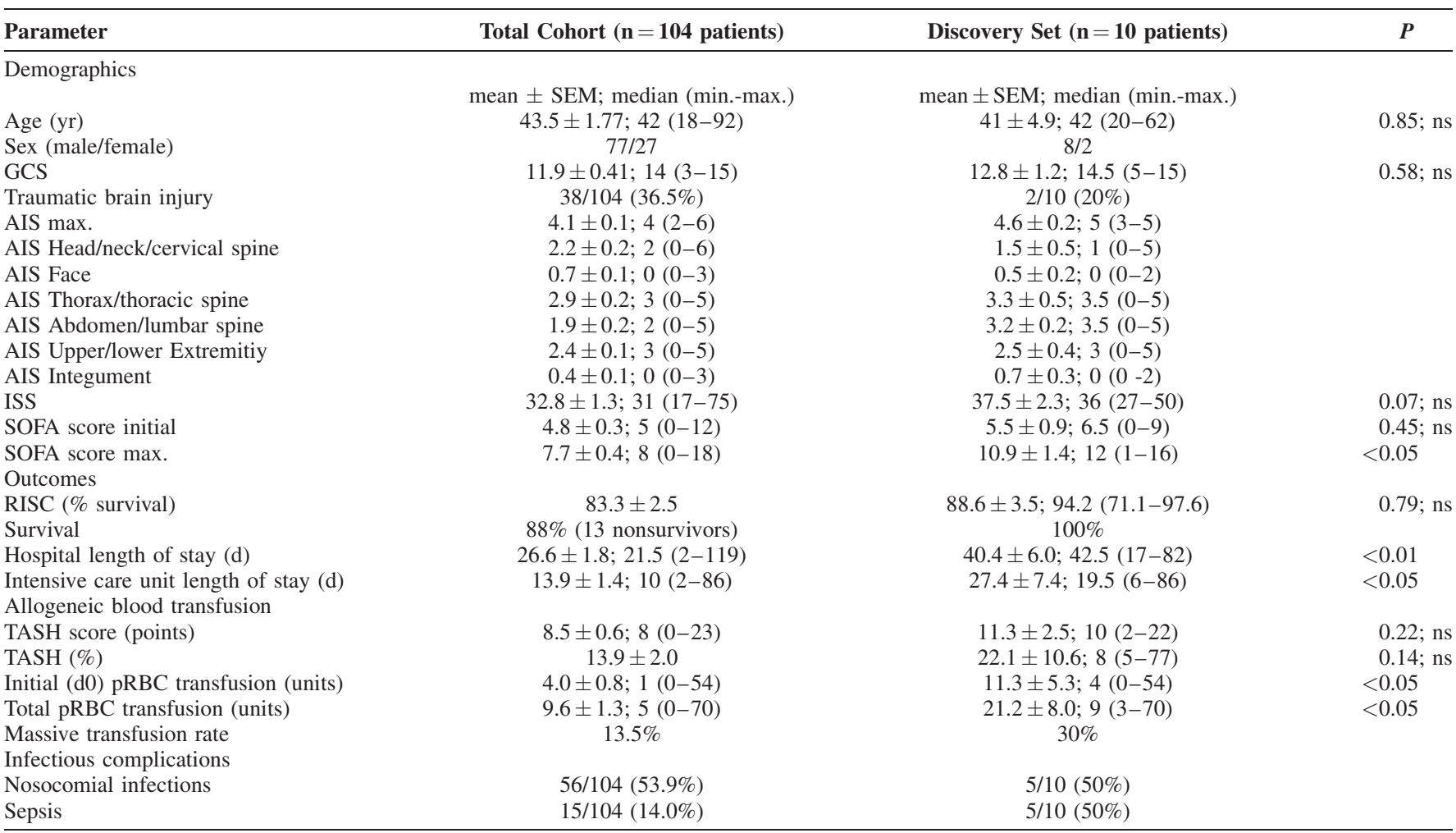

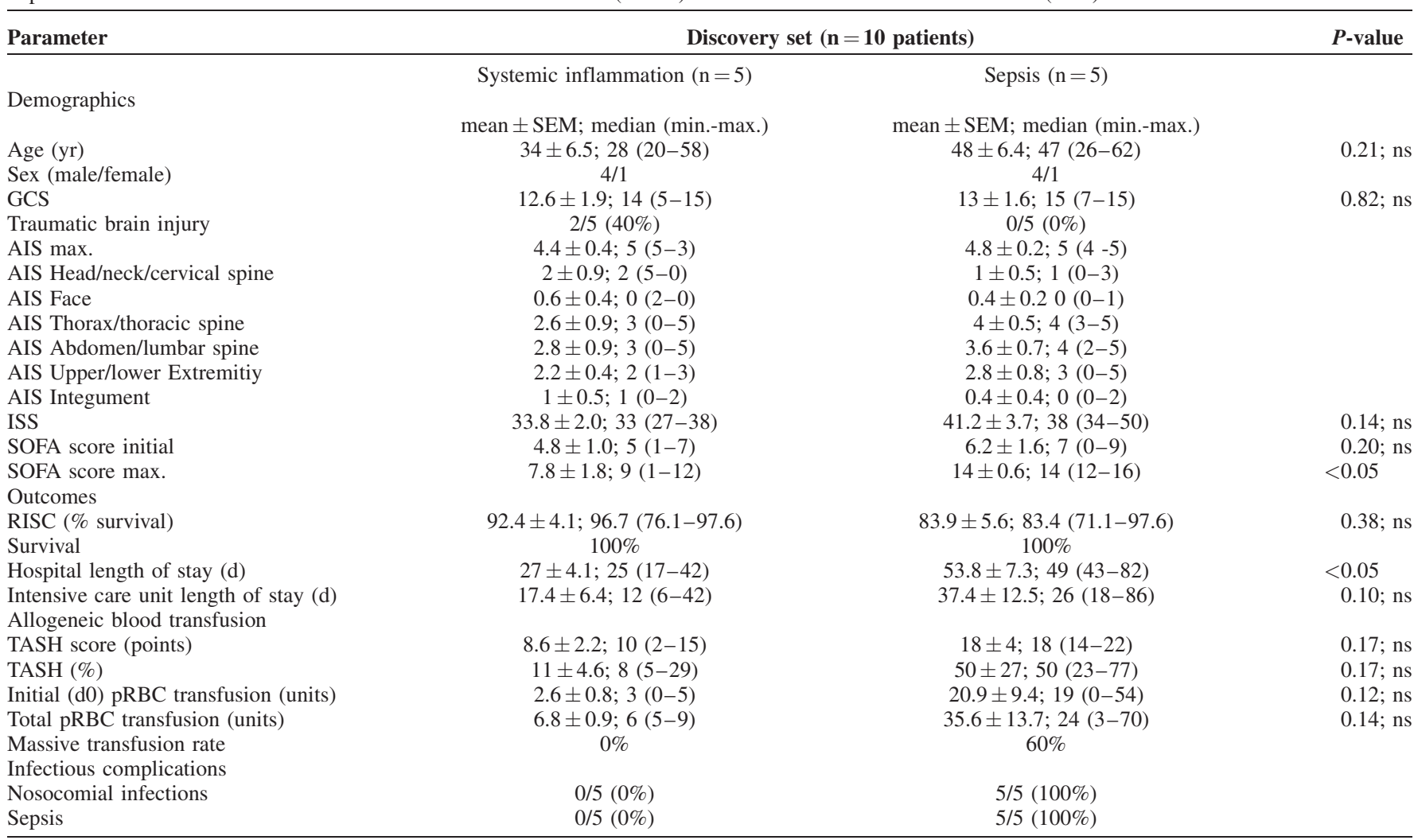



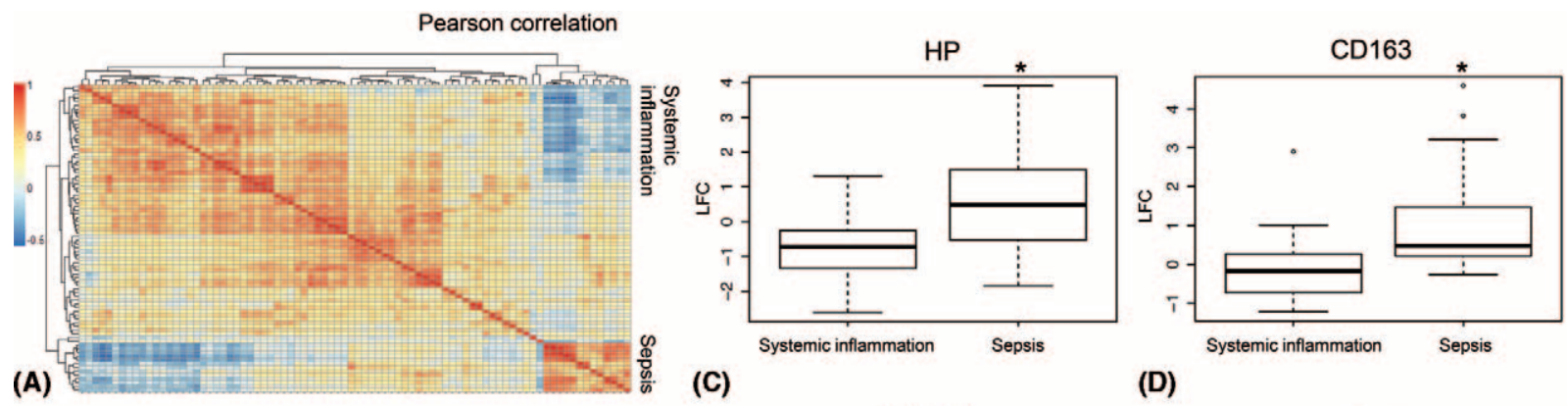

(A)

(C)

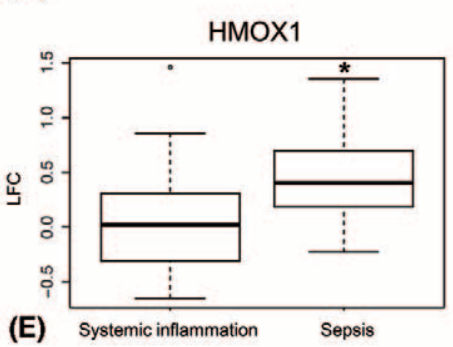

(E)

(B)
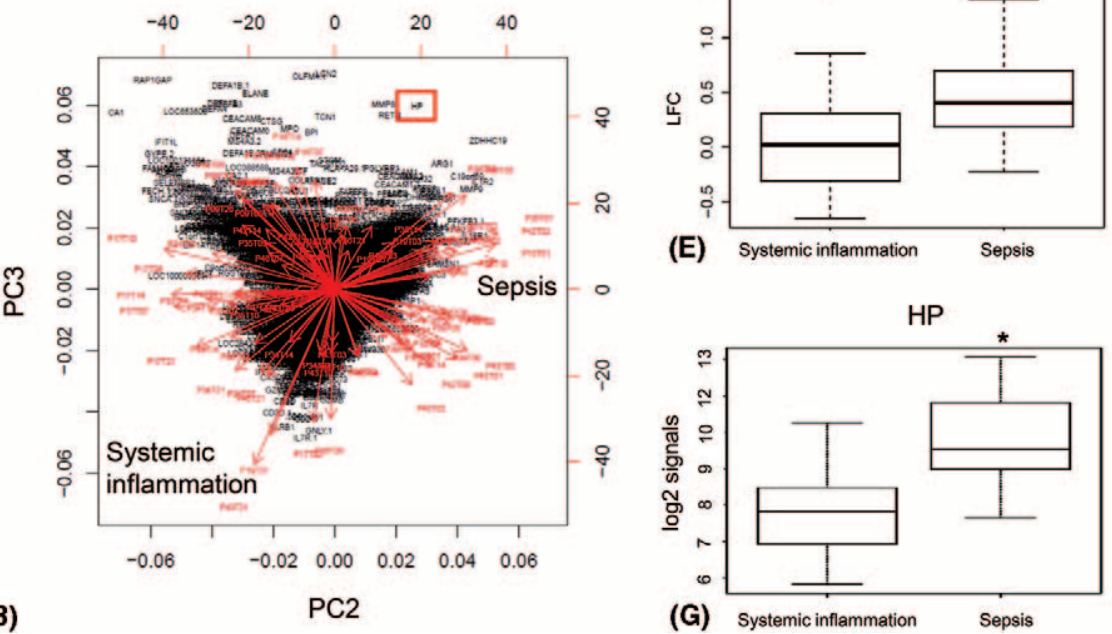

(G) Systemic inflammation Sepsis

(D)

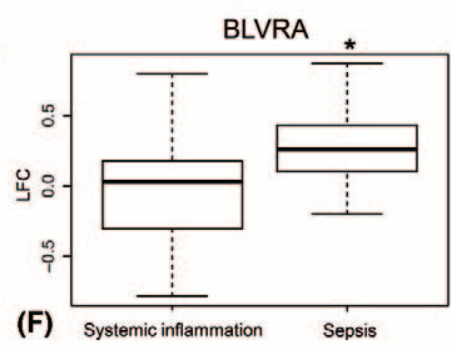

BLVRA

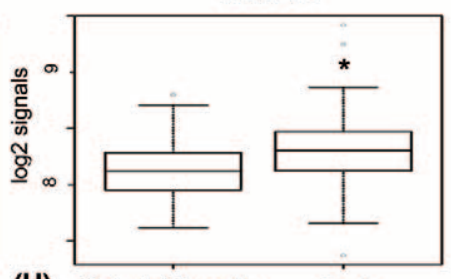

(H) Systemic inflammation Sepsis

FIGURE 1. Whole-genome analysis of circulating leukocytes from trauma patients ( $\mathrm{n}=90$ samples; 10 patients; all time points: day $0,1,2,3,5,7,10,14,21)$ who clinically either presented with systemic inflammation without infection $(n=5)$ or sepsis $(n=5)$. $(A)$ Unsupervised cluster analysis (Pearson correlation; LFC values). (B) Principal component analysis characterizing differential gene expression profiles of trauma patients. Quantification of expression (logarithmic fold change, LFC; adjusted to day 0) of HP (C), CD163 (D), HMOX1 (E), BLVRA (F), and absolute expression values for HP (G) and BLVRA (H), comparing patients with systemic inflammation or sepsis. ${ }^{*} P<0.05 ; \mathrm{NS}=$ not significant.

Moreover, principle component analysis also revealed distinct expression patterns of patients with sepsis and patients with systemic inflammation without infection, and indicated an extraordinary role of haptoglobin (HP) in patients with sepsis among all genes analyzed (Fig. 1B). In line with the findings of the discovery set, re-investigation of data sets from external, independent cohorts also identified HP among top 10 upregulated genes in patients who developed sepsis in comparison to healthy controls or patients with SIRS without infection by one-way ANOVA $\left(P<10^{-12}\right){ }^{26}$
For further analysis, expression of the key components of the heme degradation pathway HP (Fig. 1C), cluster of differentiation 163 (CD163; Fig. 1D), heme oxygenase-1 (HMOX1; Fig. 1E), and biliverdin reductase A (BLVRA; Fig. 1F) was quantified. All genes of the heme degradation pathway showed a similar pattern with significant upregulation in patients with sepsis as compared to patients with systemic inflammation without infection, as for normalization to time point $\mathrm{d} 0$ (Fig. $1 \mathrm{C}-\mathrm{F}$ ) or when comparing absolute expression values (Fig. 1G and $\mathrm{H}$ ). Furthermore, these expression

TABLE 2. Explorative Gene-Set Analysis

\begin{tabular}{|c|c|c|c|c|}
\hline Knowledge Base & Category Name & \# Hits & $\begin{array}{c}P \text {-value } \\
\text { (Fisher Exact Test) }\end{array}$ & $\begin{array}{c}\text { FDR-adjusted } \\
P \text { (Benjamini-Hochberg) }\end{array}$ \\
\hline \multicolumn{5}{|c|}{ Upregulated genes: average $\log _{2} \mathrm{FC}$ (sepsis versus systemic inflammation) $>0.5$} \\
\hline Gene Ontology CC & Hemoglobin complex & 10 & $1.60 \mathrm{E}-11$ & $6.00 \mathrm{E}-09$ \\
\hline Swiss Prot & Oxygen transport & 8 & 2.10E-08 & $3.50 \mathrm{E}-06$ \\
\hline KEGG PATHWAY & Pathogenic E. coli infection & 8 & $2.90 \mathrm{E}-06$ & 4.00E-04 \\
\hline Gene Ontology BP & Hemoglobin metabolic process & 5 & $3.00 \mathrm{E}-04$ & $5.80 \mathrm{E}-02$ \\
\hline \multicolumn{5}{|c|}{ Downregulated genes: average $\log _{2} \mathrm{FC}$ (sepsis versus systemic inflammation) $<-0.5$} \\
\hline Gene Ontology BP & Translational elongation & 26 & $5.00 \mathrm{E}-14$ & $6.90 \mathrm{E}-11$ \\
\hline Swiss Prot & Protein biosynthesis & 31 & $1.30 \mathrm{E}-12$ & $3.00 \mathrm{E}-10$ \\
\hline KEGG PATHWAY & Ribosome & 25 & $2.20 \mathrm{E}-12$ & $3.30 \mathrm{E}-10$ \\
\hline Gene Ontology BP & Defense response & 65 & $2.40 \mathrm{E}-13$ & $2.20 \mathrm{E}-10$ \\
\hline
\end{tabular}


patterns were confirmed by external validation of two independent cohorts (datasets: GPSSSI unique and GSE9960) of a recent multicohort analysis, comparing patients with sterile inflammation (SIRS/trauma) to time-matched patients with infections by wholegenome screening (Supplemental Fig. 3, http://links.lww.com/SLA/ A929). ${ }^{26-28}$

\section{Effect of Allogeneic Blood Transfusion on Clinical Outcome Parameters}

Based on the results from whole-genome screening of the discovery set, samples from all patients with complete clinicotranscriptomic coverage $(n=71 ; 517$ samples) were analyzed for gene expression patterns of HP, CD163, HMOX1, BLVRA, and BLVRB by quantitative RT-PCR normalized to the housekeeping gene ACTB $(\Delta \mathrm{Ct})$. In a first step, unsupervised clustering was applied to clinical parameters and expression of the key components of the heme degradation pathway. As shown in Fig. 2A, HP clustered with BLVRA. As to be expected, the total blood transfusion rate (pRBC-total) correlated with the Trauma Associated Severe Hemorrhage (TASH) score and the hematocrit (Fig. 2A). Among the scoring systems, the RISC score negatively correlated with the ISS, the New Injury Severity Score (NISS), and the SOFA score (Fig. 2A).
Interestingly, HP, CD163, HMOX1, BLVRA, and BLVRB collectively clustered with the ICU length of stay (ICU-LOS). Since the heme degradation pathway is activated by free heme, the initial and total amount of packed red blood cells (pRBCs) transfusion was assessed for various outcome parameters. Trauma patients who developed nosocomial infections received more units of pRBCs (initial = at the day of trauma; as well as total) than patients without infection (Fig. 2B). These differences in the amount of allogeneic blood transfusion were even more striking in sepsis patients as compared to trauma patients who did not develop secondary sepsis (Fig. 2C). In contrast, there was no difference in the initial and total volume of allogeneic blood transfusion in patients who did not survive in comparison to survivors (Fig. 2D).

In the present patient cohort, the TASH score as a measure for the risk of massive blood transfusion ( $\geq 10$ pRBC within 24 hours) matched with the actual mass transfusion rate (Table 1 ). In accordance with Fig. 2B and C, trauma patients with nosocomial infections or sepsis had significantly higher TASH scores than patients without infectious or septic complications (Fig. 2E and F).

Furthermore, the volume of allogeneic blood transfusion correlated with the ICU-LOS, as displayed in Fig. $2 \mathrm{G}$ for the total amount as well as for the initial amount of pRBC administered

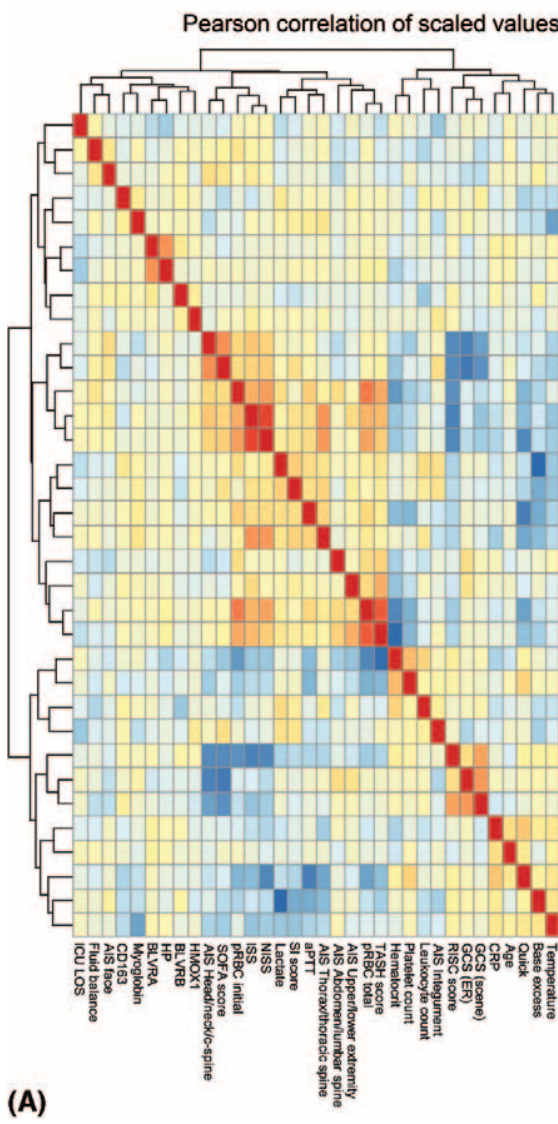

(A)

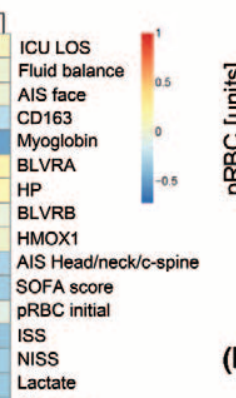

Il score

SI score

AIS Thorax/thoracic spine

AIS Abdomen/lumbar spine

AIS Upper/lower extremity

pRBC total

TASH score

Hematocrit

Platelet count

Leukocyte count

AIS Integument

RISC score

GCS (ER)

GCS (scene)

CRP

Age

Quick

Temperature

\section{(1)}

\section{.}

(6)

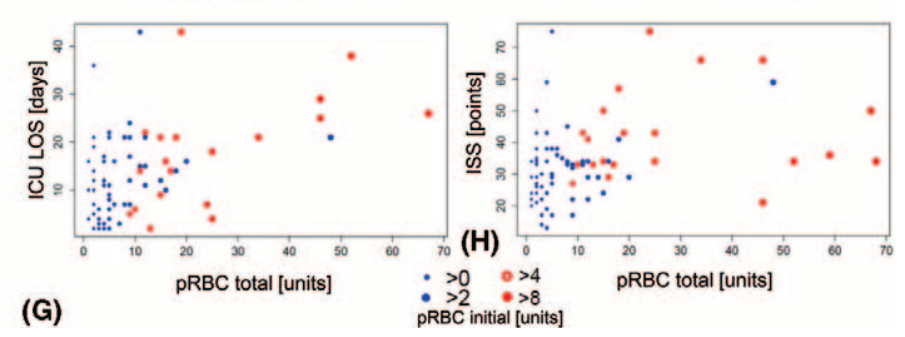

FIGURE 2. Correlation of clinical parameters of trauma patients $(n=52)$ and expression of heme degradation pathway expression ( $\triangle$ Ct of HP, CD163, HMOX1, BLVRA, and BLVRB) (A). Association of transfusion of packed red blood cells (pRBCs; total vs. initial amount $=$ at day 0 ) with nosocomial infections (B), sepsis (C), and survival (D). Comparison of TASH scores of patients with nosocomial infections $(E)$ or sepsis $(F)$ to trauma patients without infectious complications. $N=104 ;{ }^{*} P<0.05$; $N S=$ not significant. Correlation of the total amount of pRBC transfusion of with the ICU-LOS (G) and ISS (H). Initial amount of pRBC transfusion is color coded (blue represents $\leq 4$ units; red represents $>4$ units). 
(Spearman correlation coefficient $\rho$ [total $\mathrm{pRBC}]=0.55 ; \rho$ [initial $\mathrm{pRBC}]=0.39)$. However, there was also an association between the amount of $\mathrm{pRBC}$ transfusion and the ISS (Fig. 2H; $\rho$ [total $\mathrm{pRBC}]=0.43 ; \rho$ [initial $\mathrm{pRBC}]=0.44)$. Similarly, trauma patients who developed nosocomial infections or sepsis had higher ISS than patients without infections or sepsis, respectively (Supplemental Fig. 4A and B, http://links.lww.com/SLA/A929). With respect to massive blood transfusion, higher TASH scores were associated with higher ISS (Supplemental Fig. 4C, http://links.lww.com/SLA/ A929). However, there was no significant difference in the ISS in patients who did not receive allogeneic blood transfusion at all in comparison to patients who received 1 unit of $\mathrm{pRBC}$ or more (Supplemental Fig. 4D, http://links.lww.com/SLA/A929). Notably, none of the patients without allogeneic blood transfusion developed secondary sepsis.

\section{Analysis of the Expression Patterns of the Heme Degradation Pathway}

Cluster analysis of the expression of HP, CD163, HMOX1, and BLVRA as determined by microarray (discovery set; Fig. 3A) or quantitative real-time PCR (validation set; Fig. 3B) in which samples were arranged according to the clinical presentation of sepsis or systemic inflammation revealed a distinct expression pattern of HP in sepsis.

\section{Correlation of Heme Degradation Pathway Expression with Changes in the Severity of Systemic Inflammation and the Development of Sepsis}

For further correlational analysis of gene expression data with the clinical course, the SI score for assessment of the severity of systemic inflammation has been applied. As shown in Fig. 4, expression patterns of components of the heme degradation pathway followed the clinical course (SI score). Whereas shifts toward increased severity of systemic inflammation was associated with an upregulation of the heme degradation pathway components HP (Fig. 4A; ANOVA $P<10^{-10}, \rho[$ SI score, HP] $=0.44$ ) and HMOX1 (Fig. 4C), the expression of BLVRA (Fig. 4D) was downregulated with increasing SI score. These linear trends were most profound for the expression of HP (Fig. 4A). With respect to the hierarchy of the heme degradation cascade, the ratio of BLVRA and HMOX1 decreased with increasing SI score, suggesting that BLVRA becomes the rate-limiting step in higher grades of systemic inflammation (Fig. 4E). Moreover, the strongest upregulation of HP expression was found in patients who were diagnosed with sepsis (Fig. 4F), further corroborating the association between expression of the heme degradation pathway and the development of sepsis. In these patients, aggravation of systemic inflammation (positive $\Delta$ SI score) was accompanied by an upregulation of HP expression, whereas
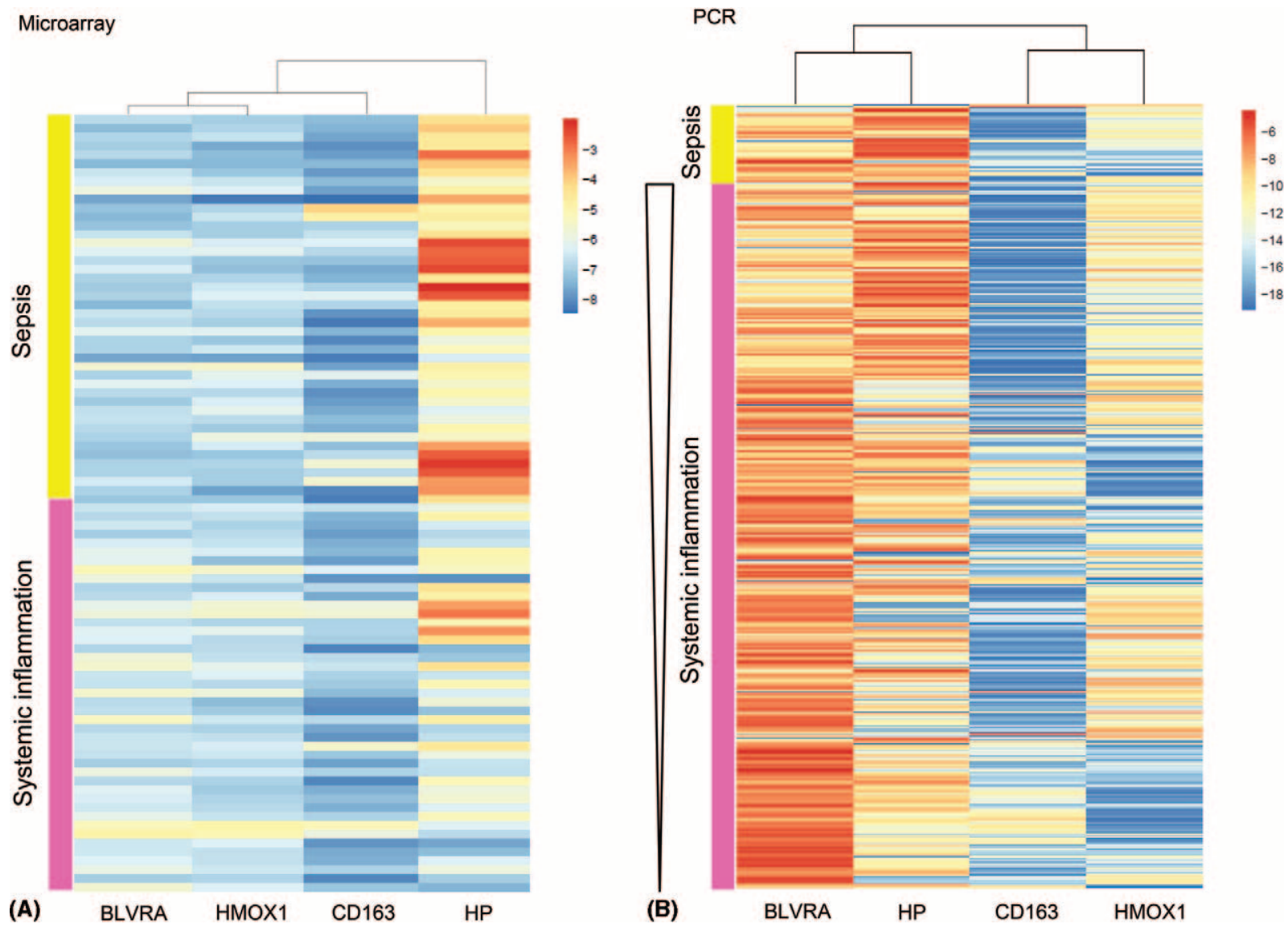

FIGURE 3. Correlation (cluster analysis) of the expression of HP, CD163, HMOX1, and BLVRA of the discovery set (A; microarray; $\mathrm{n}=90$ samples; 10 patients; all time points) and the validation set (B; quantitative RT-PCR; $\mathrm{n}=517$ samples; 71 patients; all time points) arranged by clinical presentation: sepsis vs. systemic inflammation. 

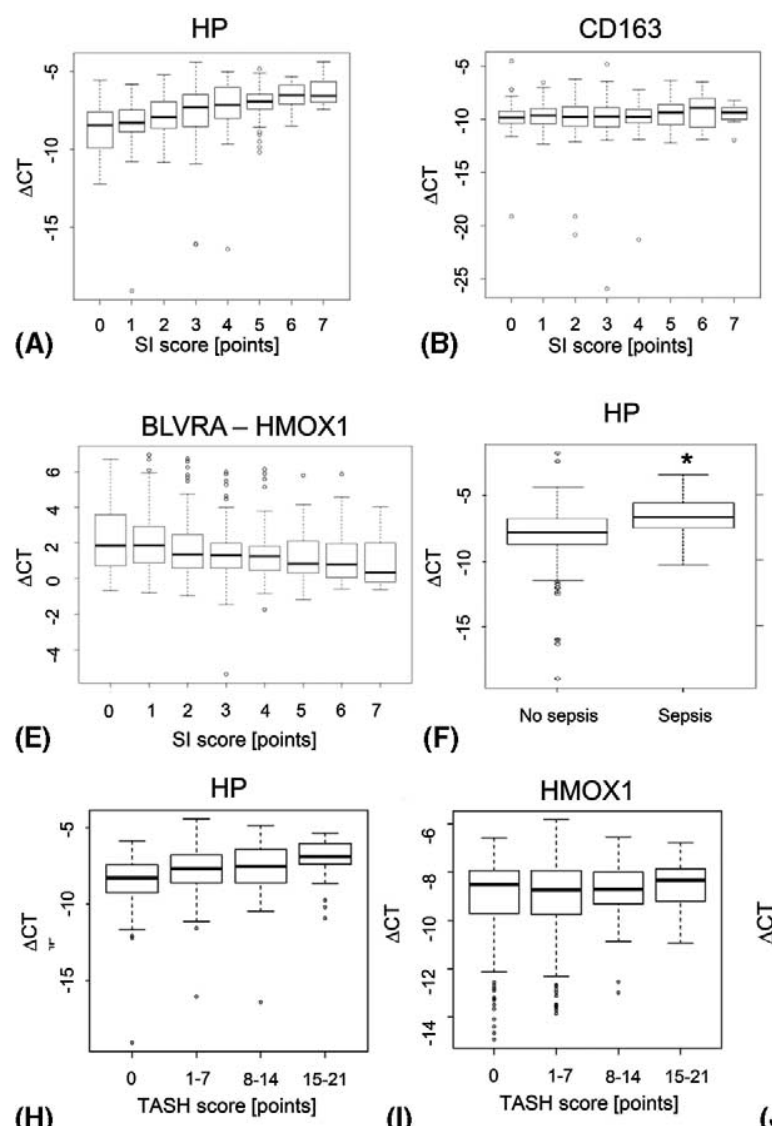

(H)

TASH score [points] (I)

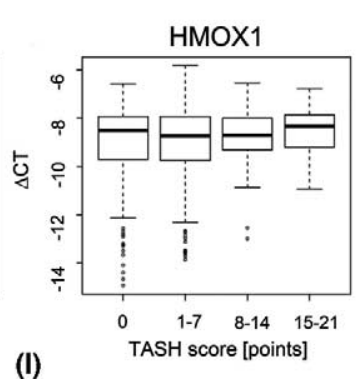

(I)
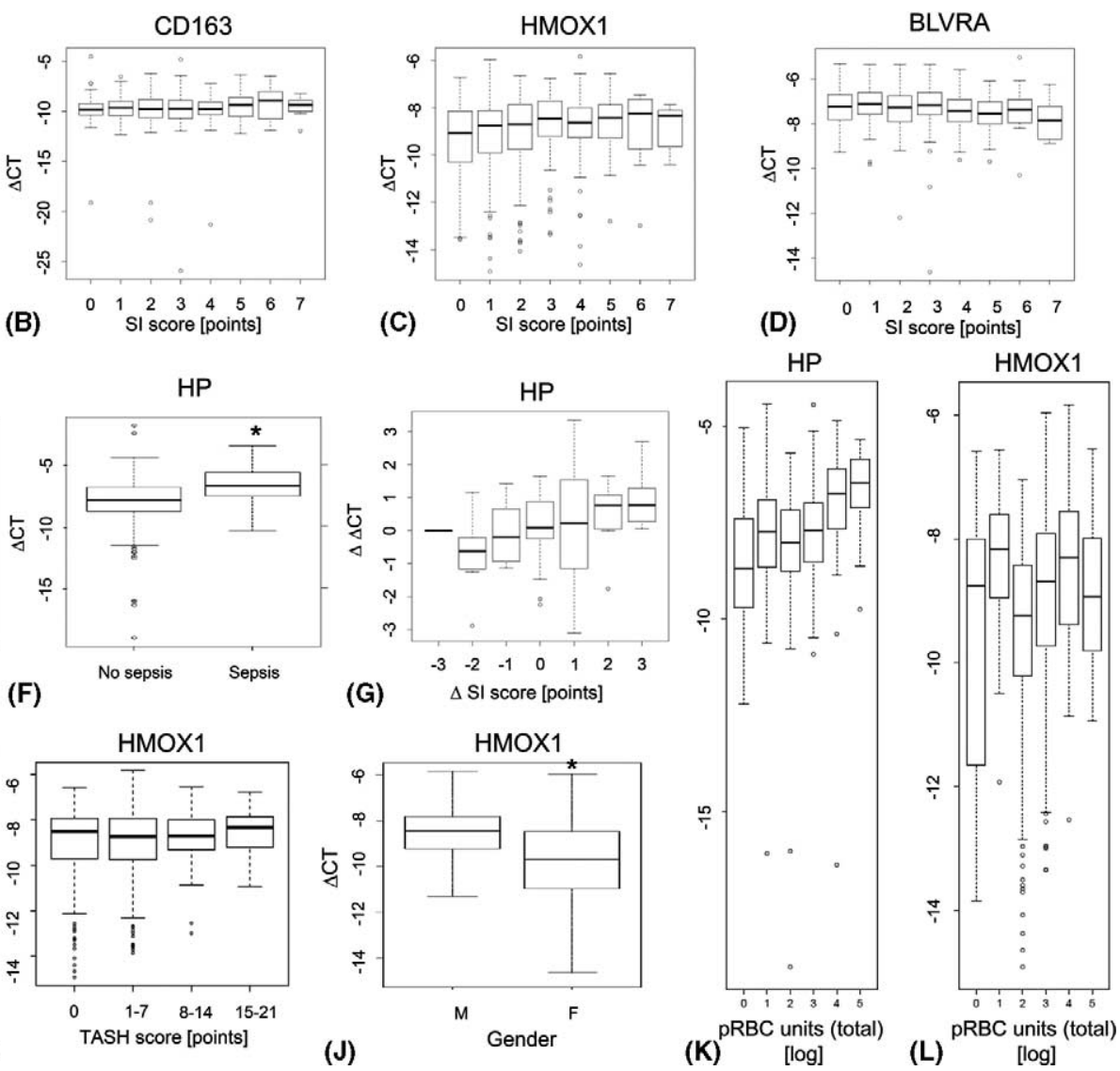

FIGURE 4. Expression ( $\triangle \mathrm{Ct}$ ) of HP (A), CD163 (B), HMOX1 (C), and BLVRA (D) in correlation with the severity of systemic inflammation (SI score). Significance, using $P<0.05$ from ANOVA and strong linear and nonlinear correlation (rho $>0.4)$ was obtained for HP. (E) Hierarchical relationship between HMOX1 and BLVRA in correlation with the SI score. (F) Expression of HP ( $\Delta$ Ct) in trauma patients with sepsis as compared to patients without sepsis. (G) Correlation of changes in $\mathrm{HP}$ expression $(\Delta \Delta \mathrm{Ct}=\mathrm{differ}-$ difference between consecutive time points) with corresponding changes in severity of systemic inflammation ( $\Delta \mathrm{SI}$ score). Association of the TASH score with the expression $(\Delta \mathrm{Ct})$ of HP $(\mathrm{H})$ and HMOX1 (I). (J) Influence of gender on HMOX expression $(\Delta \mathrm{Ct})$. Relationship between the amount of allogeneic blood transfusions (log number of total pRBCs) and the expression ( $\Delta \mathrm{Ct}$ ) of $\mathrm{HP}(\mathrm{K})$ and HMOX1 (L). $\mathrm{N} \geq 51$ patients. ${ }^{*} P<0.05$.

attenuation (negative $\Delta$ SI score) went along with downregulation of HP (Fig. 4G).

Interestingly, an increasing risk for massive transfusion (TASH score) was reflected by an upregulation of HP (Fig. 4H), whereas there were no changes in the expression of HMOX1 (Fig. 4I). As a possible explanation for the latter observation, there was a strong gender effect on the expression of HMOX1 (Fig. 4J). Similar to the TASH score, HP expression but not HMOX1 correlated with the amount of allogeneic blood transfusions (number of total pRBCs; Fig. 4K and L). In accordance, systematic, multivariate factorial analysis (MANOVA; Supplemental Table 4, http://links. lww.com/SLA/A929) showed that HP positively correlated with the rate of allogeneic blood transfusions, SI score, and sepsis. Similarly, HMOX1 expression was influenced by the total amount of pRBCs and associated with sepsis. Interestingly, these analyses showed significant time effects for BLVRA and CD163. A complete list of $P$ values is provided in Supplemental Table 4, http://links. lww.com/SLA/A929.

In further correlational analyses of heme degradation pathway expression and various mediators of inflammation (Supplemental
Fig. 5, http://links.lww.com/SLA/A929), HP expression showed a stronger positive correlation with the anti-inflammatory cytokine IL10 than with the pro-inflammatory mediators IL-8 or TNF- $\alpha$, suggesting that upregulation of the heme degradation pathway may be related to a rather anti-inflammatory phenotype.

\section{Temporal Relationship between Gene Expression and the Development of Sepsis}

In order to depict temporal changes of heme degradation pathway gene expression relative to clinical events, trajectories of septic patients were plotted in relation to the expression of the total cohort (discovery set: Fig. 5; Supplemental Fig. 6, http:// links.lww.com/SLA/A929; validation set: Supplemental Figs. 7 and 8, http://links.lww.com/SLA/A929). Although the courses were found to be highly individual for each patient, collectively, administration of allogeneic blood was followed by an upregulation of heme degradation pathway expression, and continuous increases or secondary peaks preceded the clinical diagnosis of sepsis. 

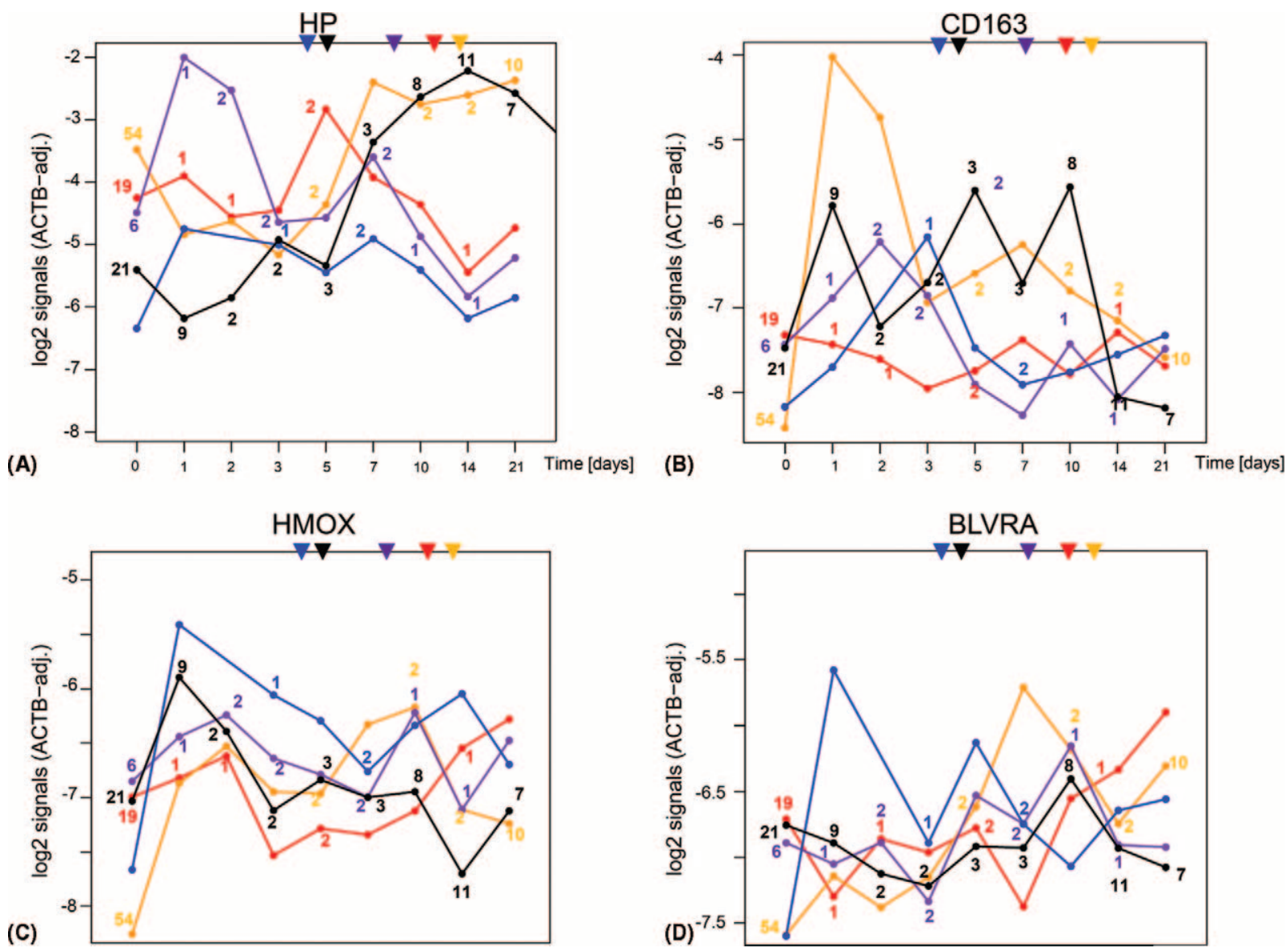

FIGURE 5. Temporal relationship of the expression of HP (A) CD163 (B), HMOX1 (C), and BLVRA (D) with the clinical course. The individual course of each patient with sepsis $(n=5)$ of the discovery set $(n=90$ samples; 10 patients $)$ are plotted as trajectories in different colors. The time points of diagnosis of sepsis are indicated by the arrows on the top of each figure. The amount of allogeneic blood transfusions (number of pRBCs) is indicated at specific time points for each patient.

\section{DISCUSSION}

The present study demonstrates for the first time that the heme degradation pathway is differentially regulated after multiple trauma and is associated with septic complications. The expression of HP in particular indicates the severity of systemic inflammation and represents a promising marker for secondary sepsis in trauma patients.

Our data suggest that the heme degradation pathway is triggered by free heme released from allogeneic blood transfusions after hemorrhagic shock, which was also associated with a significantly higher incidence of nosocomial infections and sepsis and a prolonged stay on the ICU. This is in line with several previous studies reporting that the transfusion of allogeneic blood represents an independent risk factor in critically ill patients for the development of infectious complications, MOF, and even mortality. ${ }^{1,12,29-33}$ With respect to the latter, a recent study demonstrated that only patients with a high risk of death due to traumatic hemorrhagic shock benefit from $\mathrm{pRBC}$ transfusions, whereas mortality is increased in trauma patients with a low risk of death. ${ }^{33}$ In our cohort, the expression patterns of the heme degradation pathway did not correlate with mortality, suggesting that the transcriptomic signatures are specific for the development of secondary sepsis in trauma patients but do not relate to an adverse outcome in general. Both the TASH score and amount of pRBC transfusion correlated with the development of sepsis and the HP expression pattern, corroborating the relationship between blood transfusion, regulation of the heme degradation pathway, and the development of sepsis. However, due to the clinical-translational nature of the study and multiple dependent variables, causal pathophysiologic relationships are difficult to interpret, and it remains unclear whether the changes of gene expression are secondary to blood transfusions or to the condition of hemorrhagic shock itself.

It is well established that $\mathrm{pRBCs}$ can undergo nonimmunologic hemolysis due to mechanical injury of RBCs and/or RBC storage lesions, resulting in an increased cellular fragility. Depending on the age of the pRBC product, up to $25 \%$ of the RBCs undergo nonimmunologic hemolysis within 24 hours after transfusion, resulting in a considerable amount of heme. ${ }^{34}$ In experimental sepsis, free heme has been described to play a central role by compromising the host tolerance to infection. ${ }^{35}$ Based on findings of the present study and published mechanisms of the heme degradation pathway, we conceptualize the pathophysiology in trauma as follows: free heme released from hemoglobin after allogeneic blood transfusions represents a robust proinflammatory stimulus and functions as a danger-associated molecular pattern, but it also exerts cytotoxic effects. ${ }^{36-38}$ In order to neutralize free heme, the heme-degradation pathway is activated, which may shift the balance of the immune response toward an anti-inflammatory phenotype. ${ }^{39} \mathrm{HP}$ as the 
immediate interaction partner of free heme and HMOX1 as the ratelimiting step of the enzymatic heme degradation cascade represent key components of the heme degradation pathway and are known as key regulators of the immune response. ${ }^{35,40-43}$ Prolonged dysregulation of the heme-degradation pathway may contribute to an increased susceptibility of secondary infections and the development of sepsis. On the contrary, it is conceivable that in the case of an overwhelming release of free heme, mechanisms for neutralization and degradation of heme may become saturated, resulting in remote tissue damage by circulating free heme and a reduced tolerance to infection. Experimental and clinical studies suggest that HMOX1 is the rate-limiting step of heme degradation, and that its genotypes and underlying regulatory mechanisms are associated with the outcome in sepsis. $44-47$

The particular design of the present study with purposeful selection of patients for the discovery set (secondary sepsis vs. systemic inflammation without infection) and consecutive validation of candidates in the total cohort by an independent method has been conceptualized to overcome possible masking effects by "non-selective" whole-genome screening of all patients. In contrast to our results, gene expression patterns in a similar trauma patient cohort in a previous study by the Glue grant consortium were found to be more common than different in patients with "complicated" or "uncomplicated recovery." ${ }^{13}$ As a matter of speculation, this rather unspecific distribution of patients into subgroups might have contributed to masking of differential gene expression. This assumption is supported by the fact that HP was also found among the genes whose expression increased the most in the "genomic storm" study. ${ }^{13}$ In fact, similar correlations of heme degradation pathway expression with clinical variables as in our study were found reanalyzing the publicly available Glue grant data set (eg, correlation of HP expression with volume of allogeneic blood transfusions, incidence of nosocomial infections and SSI, or ICU-LOS; data not shown) and additional data sets of two independent cohorts that are part of a recent multicohort analysis, comparing patients with sterile inflammation to sepsis. ${ }^{13,26-28}$

In our cohort as well as in external data sets, HP expression has been consistently identified as a promising marker for sepsis in trauma patients, which may even be superior to and more specific than serum bilirubin, as HP expression precedes the generation of bilirubin, and high bilirubin levels can not only be caused by increased heme degradation but also by impaired hepatic secretion. ${ }^{48}$

Our data indicate that an integrated clinico-transcriptomic approach facilitates to distinguish between systemic inflammation without infection and sepsis after trauma, in accordance with previous studies in trauma and nontrauma patients with sepsis. ${ }^{26,49-51}$ With respect to the impact on the treatment strategy of trauma patients, our findings imply that patients who received blood transfusions should be closely monitored regarding the development of septic complications using specific markers. In future, routine evaluation of trauma patients may ideally comprise integrated assessment of clinical, laboratory, proteomic, and transcriptomic parameters, on the basis of which treatment algorithms, including timing of secondary surgical interventions as well as operative techniques to be applied, could be adapted to the individual risk profile. The expression patterns of the heme degradation pathway in leukocytes, with HP in particular, may help assess the host immune status and response and stratify the individual risk of trauma patients for infectious complications and sepsis.

\section{REFERENCES}

1. Wafaisade A, Lefering R, Bouillon B, et al. Epidemiology and risk factors of sepsis after multiple trauma: an analysis of 29,829 patients from the Trauma Registry of the German Society for Trauma Surgery. Crit Care Med. 2011;39:621-628
2. MacKenzie EJ. Epidemiology of injuries: current trends and future challenges. Epidemiol Rev. 2000;22:112-119.

3. Mock CN, Jurkovich GJ, nii-Amon-Kotei D, et al. Trauma mortality patterns in three nations at different economic levels: implications for global trauma system development. J Trauma. 1998;44:804-812; discussion 812-814.

4. Hirsiger S, Simmen HP, Werner CM, et al. Danger signals activating the immune response after trauma. Mediators Inflamm. 2012;2012:315941.

5. Gentile LF, Cuenca AG, Efron PA, et al. Persistent inflammation and immunosuppression: a common syndrome and new horizon for surgical intensive care. J Trauma Acute Care Surg. 2012;72:1491-1501.

6. Faist E, Baue AE, Dittmer H, et al. Multiple organ failure in polytrauma patients. J Trauma. 1983;23:775-787.

7. Rotondo MF, Schwab CW, McGonigal MD, et al. 'Damage control': an approach for improved survival in exsanguinating penetrating abdominal injury. J Trauma. 1993;35:375-382; discussion 382-383.

8. Spahn DR, Bouillon B, Cerny V, et al. Management of bleeding and coagulopathy following major trauma: an updated European guideline. Crit Care. 2013; 17:R76.

9. Pape HC, Giannoudis P, Krettek C. The timing of fracture treatment in polytrauma patients: relevance of damage control orthopedic surgery. Am J Surg. 2002;183:622-629.

10. Moore FA, Sauaia A, Moore EE, et al. Postinjury multiple organ failure: a bimodal phenomenon. J Trauma. 1996;40:501-510; discussion 510-512.

11. Bone RC. Toward a theory regarding the pathogenesis of the systemic inflammatory response syndrome: what we do and do not know about cytokine regulation. Crit Care Med. 1996;24:163-172.

12. Lord JM, Midwinter MJ, Chen YF, et al. The systemic immune response to trauma: an overview of pathophysiology and treatment. Lancet. 2014:384:1455-1465.

13. Xiao W, Mindrinos MN, Seok J, et al. A genomic storm in critically injured humans. J Exp Med. 2011;208:2581-2590.

14. Rittirsch D, Flierl MA, Ward PA. Harmful molecular mechanisms in sepsis. Nat Rev Immunol. 2008;8:776-787.

15. Lendemans S, Ruchholtz S. German Society of Trauma S. [S3 guideline on treatment of polytrauma/severe injuries. Trauma room care]. Unfallchirurg. 2012;115:14-21.

16. Vincent JL, Moreno R, Takala J, et al. The SOFA (Sepsis-related Organ Failure Assessment) score to describe organ dysfunction/failure. On behalf of the Working Group on Sepsis-Related Problems of the European Society of Intensive Care Medicine. Intensive Care Med. 1996;22:707-710.

17. Bone RC, Balk RA, Cerra FB, et al. Definitions for sepsis and organ failure and guidelines for the use of innovative therapies in sepsis. The ACCP/SCCM Consensus Conference Committee. American College of Chest Physicians/ Society of Critical Care Medicine. Chest. 1992;101:1644-1655.

18. Levy MM, Fink MP, Marshall JC, et al. 2001 SCCM/ESICM/ACCP/ATS/SIS International Sepsis Definitions Conference. Crit Care Med. 2003;31:12501256.

19. Reinhart K, Brunkhorst FM, Bone HG, et al. Prevention, diagnosis, therapy and follow-up care of sepsis: 1st revision of S-2k guidelines of the German Sepsis Society (Deutsche Sepsis-Gesellschaft e.V. (DSG)) and the German Interdisciplinary Association of Intensive Care and Emergency Medicine (Deutsche Interdisziplinare Vereinigung fur Intensiv- und Notfallmedizin (DIVI)). Ger Med Sci. 2010;8:Doc14.

20. Rangel-Frausto MS, Pittet D, Costigan M, et al. The natural history of the systemic inflammatory response syndrome (SIRS). A prospective study. JAMA. 1995;273:117-123.

21. Napolitano LM, Ferrer T, McCarter RJ Jr, et al. Systemic inflammatory response syndrome score at admission independently predicts mortality and length of stay in trauma patients. J Trauma. 2000;49:647-652; discussion $652-653$.

22. Malone DL, Kuhls D, Napolitano LM, et al. Back to basics: validation of the admission systemic inflammatory response syndrome score in predicting outcome in trauma. $J$ Trauma. 2001;51:458-463.

23. Talmor M, Hydo L, Barie PS. Relationship of systemic inflammatory response syndrome to organ dysfunction, length of stay, and mortality in critical surgical illness: effect of intensive care unit resuscitation. Arch Surg. 1999;134:81-87.

24. Kaukonen KM, Bailey M, Pilcher D, et al. Systemic inflammatory response syndrome criteria in defining severe sepsis. N Engl J Med. 2015;372:1629-1638.

25. Gentleman RC, Carey VJ, Bates DM, et al. Bioconductor: open software development for computational biology and bioinformatics. Genome Biol. 2004;5:R80.

26. Sweeney TE, Shidham A, Wong HR, et al. A comprehensive time-coursebased multicohort analysis of sepsis and sterile inflammation reveals a robust diagnostic gene set. Sci Transl Med. 2015;7:287ra71. 
27. Wong HR, Cvijanovich N, Allen GL, et al. Genomic expression profiling across the pediatric systemic inflammatory response syndrome, sepsis, and septic shock spectrum. Crit Care Med. 2009;37:1558-1566.

28. Tang BM, McLean AS, Dawes IW, et al. Gene-expression profiling of peripheral blood mononuclear cells in sepsis. Crit Care Med. 2009;37: $882-888$.

29. Beale E, Zhu J, Chan L, et al. Blood transfusion in critically injured patients: a prospective study. Injury. 2006;37:455-465.

30. Croce MA, Fabian TC, Waddle-Smith L, et al. Identification of early predictors for post-traumatic pneumonia. Am Surg. 2001;67:105-110.

31. Dunne JR, Malone DL, Tracy JK, et al. Allogenic blood transfusion in the first 24 hours after trauma is associated with increased systemic inflammatory response syndrome (SIRS) and death. Surg Infect (Larchmt). 2004;5:395-404.

32. Flohe S, Kobbe P, Nast-Kolb D. Immunological reactions secondary to blood transfusion. Injury. 2007;38:1405-1408.

33. Perel P, Clayton T, Altman DG, et al. Red blood cell transfusion and mortality in trauma patients: risk-stratified analysis of an observational study. PLoS Med. 2014;11:e1001664.

34. Aubron C, Nichol A, Cooper DJ, et al. Age of red blood cells and transfusion in critically ill patients. Ann Intensive Care. 2013;3:2.

35. Larsen R, Gozzelino R, Jeney V, et al. A central role for free heme in the pathogenesis of severe sepsis. Sci Transl Med. 2010;2:51ra71.

36. Fernandez PL, Dutra FF, Alves L, et al. Heme amplifies the innate immune response to microbial molecules through spleen tyrosine kinase (Syk)-dependent reactive oxygen species generation. J Biol Chem. 2010;285:32844-32851.

37. Figueiredo RT, Fernandez PL, Mourao-Sa DS, et al. Characterization of heme as activator of Toll-like receptor 4. J Biol Chem. 2007;282:20221-20229.

38. Larsen R, Gouveia Z, Soares MP, et al. Heme cytotoxicity and the pathogenesis of immune-mediated inflammatory diseases. Front Pharmacol. 2012;3:77.

39. Dutra FF, Bozza MT. Heme on innate immunity and inflammation. Front Pharmacol. 2014;5:115.

40. Wegiel B, Gallo D, Csizmadia E, et al. Biliverdin inhibits Toll-like receptor-4 (TLR4) expression through nitric oxide-dependent nuclear translocation of biliverdin reductase. Proc Natl Acad Sci U S A. 2011;108:18849-18854.

41. Buechler C, Ritter M, Orso E, et al. Regulation of scavenger receptor CD163 expression in human monocytes and macrophages by pro- and antiinflammatory stimuli. J Leukoc Biol. 2000;67:97-103.
42. Lee TS, Chau LY. Heme oxygenase-1 mediates the anti-inflammatory effect of interleukin-10 in mice. Nat Med. 2002;8:240-246.

43. Huntoon KM, Wang Y, Eppolito CA, et al. The acute phase protein haptoglobin regulates host immunity. J Leukoc Biol. 2008;84:170-181.

44. Tenhunen R, Marver HS, Schmid R. The enzymatic conversion of heme to bilirubin by microsomal heme oxygenase. Proc Natl Acad Sci U SA. 1968;61:748-755.

45. Thomsen JH, Etzerodt A, Svendsen P, et al. The haptoglobin-CD163-heme oxygenase-1 pathway for hemoglobin scavenging. Oxid Med Cell Longev. 2013;2013:523652.

46. Kramer M, Sponholz C, Slaba M, et al. Alternative 5' untranslated regions are involved in expression regulation of human heme oxygenase-1. PLoS One. 2013;8:e77224.

47. Sponholz C, Huse K, Kramer M, et al. Gene polymorphisms in the heme degradation pathway and outcome of severe human sepsis. Shock. 2012;38:459-465.

48. Patel JJ, Taneja A, Niccum D, et al. The association of serum bilirubin levels on the outcomes of severe sepsis. J Intensive Care Med. 2015;30:23-29.

49. Langley RJ, Tsalik EL, van Velkinburgh JC, et al. An integrated clinicometabolomic model improves prediction of death in sepsis. Sci Transl Med. 2013;5:195ra95.

50. Langley RJ, Tipper JL, Bruse S, et al. Integrative "omic" analysis of experimental bacteremia identifies a metabolic signature that distinguishes human sepsis from systemic inflammatory response syndromes. Am J Respir Crit Care Med. 2014;190:445-455.

51. Lindig S, Quickert S, Vodovotz Y, et al. Age-independent co-expression of antimicrobial gene clusters in the blood of septic patients. Int J Antimicrob Agents. 2013;42(Suppl):S2-S7.

52. Du P, Kibbe WA, Lin SM. lumi: a pipeline for processing Illumina microarray. Bioinformatics. 2008;24:1547-1548.

53. Feng G, Shaw P, Rosen ST, et al. Using the bioconductor GeneAnswers package to interpret gene lists. Methods Mol Biol. 2012;802:101-112.

54. Croft D, Mundo AF, Haw R, et al. The Reactome pathway knowledgebase. Nucleic Acids Res. 2014;42:D472-D477.

55. Ashburner M, Ball CA, Blake JA, et al. Gene ontology: tool for the unification of biology. The Gene Ontology Consortium. Nat Genet. 2000;25:25-29.

56. Subramanian A, Tamayo P, Mootha VK, et al. Gene set enrichment analysis: a knowledge-based approach for interpreting genome-wide expression profiles. Proc Natl Acad Sci U S A. 2005;102:15545-15550. 